\title{
$O$ acometimento renal na hipertensão arterial e diabetes mellitus tipo 2: como identificar e prevenir A visão do nefrologista
}

\author{
The renal involvement in hypertension and type 2 diabetes mellitus: \\ how to identify and to prevent? The vision of the nephrologist
}

José Mauro Vieira Júnior*

José Hermógenes Rocco Suassuna

\section{Resumo}

Cerca de $30 \%$ dos pacientes com diabetes mellitus desenvolvem nefropatia diabética, especialmente em associação com hipertensão arterial. Além disso, essa patologia responde por 30-40\% dos pacientes em diálise crônica. A doença renal crônica, mesmo em estágios menos graves, é um importante determinante de desfechos adversos em pacientes hipertensos e diabéticos, sendo atualmente considerada um dos mais significativos fatores não tradicionais de risco cardiovascular. Entretanto, vale ressaltar que o risco cardiovascular também aumenta com a perda da filtração glomerular, de forma proporcional e independente. Ao contrário do DM tipo I, clinicamente a nefropatia diabética no tipo II pode caracterizar-se por microalbuminúria no momento do diagnóstico, perda da função renal sem microalbuminúria, macroalbuminúria sem microalbuminúria prévia, ou hipertensão grave e patologia vascular precoce. Os achados histopatológicos são heterogêneos, apresentado uma mistura de glomerulosclerose diabética e nefrosclerose hipertensiva, mas também outras lesões vasculares e isquemia glomerular. A base para a prevenção e tratamento da nefropatia diabética e suas comorbidades cardiovasculares está no 1) controle metabólico intensivo; 2) controle pressórico rigoroso e 3) bloqueio do sistema renina-angiotensina. Diversos estudos sugerem que esta última intervenção parece ter efeitos que independem e ultrapassam o controle pressórico. O bloqueio da angiotensina determina diminuição da proteinúria, da hipetensão e hipertrofia glomerular, além de ter efeitos anti-inflamatório e antifibrótico. Novos tratamentos da nefropatia diabética com substâncias antioxidantes e anti-inflamatórios/ antifibróticos vêm sendo propostos, com resultados encorajadores.

Descritores: Diabetes mellitus; Insuficiência renal crônica; Proteinúria; Hipertensão. 


\section{Abstract}

Approximately $30 \%$ of diabetic patients develop diabetic nephropathy, particularly associated with arterial hypertension. Moreover, this disease accounts for $30-40 \%$ of patients undergoing chronic dialysis. Chronic renal disease, even if mild, is clearly associated with unfavorable outcomes in diabetic and hypertensive patients. It is actually considered one of the most important nontraditional cardiovascular risk factors. However, it is noteworthy that the cardiovascular risk is independently related to the degree of the renal dysfunction. In contrast to type I DM, type II-associated diabetic nephropathy may be characterized by microalbuminuria at onset, loss of function without microalbuminuria, albuminuria without preceding microalbuminuria, or early and severe vascular disease. The renal pathological findings are heterogeneous, comprising from a mixture of glomerulosclerosis and hypertensive nephroesclerosis to vascular lesions and glomerular ischemia. The recommended approach to prevent and treat diabetic nephropathy is based on: 1) intensive metabolic control; 2) rigorous blood pressure control, and 3) renin-angiotensin system (RAS) blockade. Several studies suggest that the RAS blockade might have blood pressure-independent beneficial effects. RAS blockade leads to proteinuria decrease, attenuation of glomerular hypertrophy and hyperfiltration, along with antiinflamatory and antifibrotic effects. Newer treatments for diabetic nephropathy based on antioxidant and antifibrotic drugs have been described, with encouraging early results.

Keywords: Diabetes mellitus; Renal insufficiency chronic; Proteinuria; Hypertension.

\section{Epidemiologia e o impacto da doença renal crônica no contexto de hipertensão arterial e diabetes mellitus}

Atualmente, as doenças crônicas não transmissíveis (DCNTs) são o principal problema global de saúde. Das 57 milhões de mortes ocorridas no mundo em 2008 63\% decorreram por DCNTs, sendo a imensa maioria em países de baixa e média renda, como o Brasil. ${ }^{1}$ A doença renal crônica (DRC) sempre esteve ausente dessas estatísticas. As nefropatias só eram notadas em sua vertente mais dramática - a DRC estabelecida, antigamente chamada de terminal -, que se associa aos elevados custos econômicos e sociais da diálise crônica e do transplante renal. Várias seriam as razões para essa "invisibilidade". Na DRC predominam os casos oligo ou assintomáticos. Existe, por conseguinte, dependência fundamental de testes laboratoriais para o diagnóstico que, além disso, não demonstram relação linear com a gravidade do acometimento renal. Por fim, o sistema de classificação internacional de doenças (CID) só passou a incluir estágios não terminais da DRC a partir de 2010 (a versão usada no Brasil ainda é de 2008).

Outro problema é a associação frequente com condições de maior visibilidade, que desviam a preocupação em relação aos rins. Por exemplo, alterações da função renal são frequentes em pacientes com hipertensão e cardiopatia isquêmica, especialmente em portadores de diabetes. De fato, globalmente a grande maioria dos casos de DRC acontece em associação com hipertensão, diabetes, doenças cardiovasculares, envelhecimento e obesidade (figura 1). $\mathrm{O}$ acometimento renal pelo diabetes

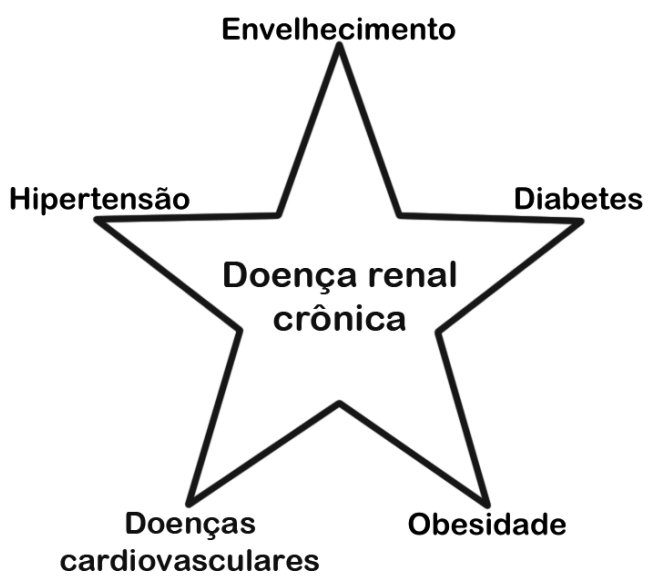

Figura 1. Principais associações clínicas da doença renal crônica 
responde por $40 \%$ dos casos incidentes de diálise nos Estados Unidos, e no Brasil rivaliza com a hipertensão, acometendo 30\% dos pacientes que iniciam tratamento dialítico. Estes pacientes sofrem um risco adicional de morte de $20 \%$ no primeiro ano de diálise, principalmente por causas cardiovasculares. ${ }^{2}$ Em outras palavras, cerca de $30 \%$ dos pacientes com diabetes desenvolvem nefropatia diabética, especialmente em associação com hipertensão. A DRC é um importante determinante de desfechos adversos em pacientes hipertensos e diabéticos, sendo atualmente considerada um dos mais significativos fatores não tradicionais de risco cardiovascular. ${ }^{3} \mathrm{O}$ risco cardiovascular de um paciente diabético do tipo 2 é considerado equivalente ao de um não diabético com infarto prévio, ${ }^{4}$ mas esse risco aumenta ainda mais com o advento da nefropatia diabética. ${ }^{5}$ Por fim, indivíduos com DRC, com frequência, são acometidos por doenças cardiovasculares, em particular aqueles que também sofrem de diabetes. ${ }^{6}$ Como evidências epidemiológicas indicam que a prevalência desses fatores de risco aumenta globalmente, a expectativa é que também cresça o impacto das doenças renais. ${ }^{7}$

\section{Formas de acometimento renal e como identificá-las}

A doença renal associada ao diabetes ocorre no contexto de hipertensão arterial como condi- ção subjacente. A patogênese do envolvimento renal no diabetes é melhor conhecida nos casos de diabetes do tipo 1 (DM1). Acredita-se que os eventos hemodinâmicos iniciais de hiperperfusão, hipertensão e hiperfiltração glomerular causam vazamento glomerular de macromoléculas, principalmente de albumina, que resulta em espessamento da membrana basal glomerular, hipertrofia glomerular, expansão mesangial, lesão podocitária e glomerulosclerose. ${ }^{8}$ Estes fenômenos (figura 2) expressam-se clinicamente por albuminúria (inicialmente intermitente e de pequena monta e, depois, crescente e persistente) e por diminuição da taxa de filtração glomerular (TFG). Classicamente, no DM1 é possível identificar uma sequência de fases (figura 2), da eventual progressão à fase de doença renal estabelecida. ${ }^{9}$

Este padrão sequencial é pouco evidente no diabetes tipo 2 (DM2). Os achados histopatológicos são heterogêneos, apresentando uma mistura de aspectos. Existe glomerulosclerose diabética e nefrosclerose hipertensiva, mas também outras lesões vasculares, isquemia glomerular e uma variedade de lesões glomerulares superpostas. ${ }^{10}$ Clinicamente pode haver microalbuminúria no momento do diagnóstico, perda da função renal sem microalbuminúria, macroalbuminúria sem microalbuminúria prévia e hipertensão e patologia vascular precoces. Além da nefropatia, a albuminúria também

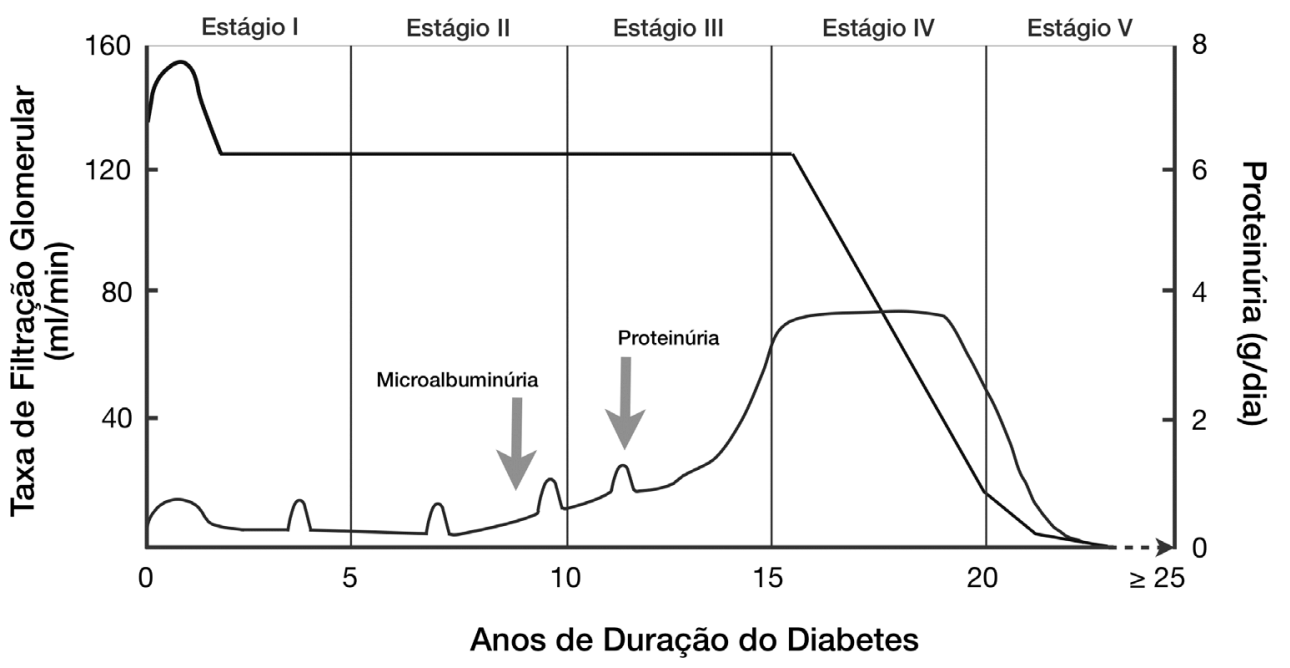

Figura 2. Estágios da nefropatia diabética 
pode indicar disfunção endotelial sistêmica, o que explica sua associação com doenças cardiovasculares. ${ }^{5}$ Vale ressaltar que o risco cardiovascular também aumenta com a perda da TFG, de forma proporcional e independente. ${ }^{8}$

A explicação fisiopatológica para a associação entre disfunção renal e risco cardiovascular não é simples. ${ }^{8}$ É possível que vários fatores atuem de forma complementar (lesão vascular direta por hipertensão e hiperglicemia, rigidez vascular, disfunção endotelial, inflamação, dislipidemia, estresse oxidativo, deficiência de vitamina $\mathrm{D}$ etc.), o que justificaria uma abordagem terapêutica multifatorial (ver adiante).

O diagnóstico das nefropatias baseia-se na observação de comprometimento funcional ou estrutural renal. Identifica-se a disfunção renal pela diminuição da TFG, medida diretamente ou estimada mediante fórmulas, principalmente as derivadas da creatinina sérica. Essas equações utilizam parâmetros como idade, gênero, etnia e tamanho corporal, que são determinantes não renais da creatinina sérica, para aumentar a precisão da aferição da função renal. É interessante notar que a TFG estimada não está sujeita aos erros de coleta de urina que prejudicam o uso do clearance de creatinina e, quando inferior a $60 \mathrm{ml} / \mathrm{min}$, apresenta correlação estreita com o risco futuro de eventos cardiovasculares. ${ }^{11}$

O dano estrutural é identificado diretamente (métodos de imagem, biópsia renal, achado cirúrgico) ou por marcadores como proteinúria, hematúria de origem renal ou outras anormalidades do sedimento urinário. Do ponto de vista prático a albuminúria pode ser detectada de forma semiquantitativa, em fita tipo dipstick, ou por ensaios imunológicos, sensíveis na faixa de microgramas por decilitro. É importante ressaltar que, em geral, tratam-se de técnicas simples, relativamente baratas e amplamente disponíveis.

\section{Proteção renal no hipertenso diabético}

\section{Controle pressórico}

Na nefropatia diabética é claro o papel do controle da pressão arterial (PA) sistêmica na atenuação da progressão renal, assim como a importância da angiotensina como mediador das lesões renais, com destaque para o fato de que suas ações renais vão além do controle pressórico. ${ }^{12}$ Por outro lado, é consenso que a busca do controle pressórico ultrapassa discussões relativas a diferentes classes de drogas. Dito de outra maneira, não cabe discutir o benefício de determinada classe de droga sem que antes se assegure o controle pressórico rigoroso nos pacientes diabéticos hipertensos.

Estudos experimentais com modelos murinos de DM demonstraram o benefício do bloqueio do sistema renina-angiotensinaaldosterona (SRAA) com inibidores da enzima conversora da angiotensina (IECAs) ou bloqueadores dos receptores da angiotensina (BRAs). A histologia renal é claramente protegida e ocorre diminuição da albuminúria e atenuação da progressão da disfunção renal. Os mecanismos apontados, muitos independentes do controle pressórico, incluem diminuição da produção de citocinas pró-fibróticas, diminuição de processos inflamatórios não imunes (por exemplo, influxo tecidual de células inflamatórias) e diminuição do stress oxidativo, dentre outros.

Estudos randomizados que compararam o uso de IECAs ou BRAs em pacientes diabéticos hipertensos, contra o mesmo grau de controle pressórico no grupo controle, ${ }^{13-15}$ observaram redução da macroalbuminúria, diminuição na evolução de micro para macroalbuminúria e diminuição do risco de duplicar o valor da creatinina sérica. Alguns desses estudos demonstraram melhora de desfechos mais robustos como a necessidade de tratamento dialítico. Essas observações formam a base para a recomendação atual, que é buscar a dose mais alta tolerada de um IECA ou BRA em monoterapia. Não parece haver superioridade de uma classe sobre a outra e drogas de classes adicionais podem e devem ser usadas na frequente situação de HA resistente.

Para pacientes diabéticos com DRC (definida como TFG $<60 \mathrm{ml} / \mathrm{min}$ ), a American Diabetes Association e a National Kidney Foundation compartilham o alvo da PA sis- 
tólica menor que $130 \mathrm{mmHg}$ e PA diastólica menor que $80 \mathrm{mmHg}$. A observância a esses alvos correlaciona-se com proteção de eventos cardiovasculares e nefroproteção. Por exemplo, no estudo ACCORD houve redução do risco de doença cerebrovascular em pacientes com DM2 tratados com alvo de PA sistólica em 120 $\mathrm{mmHg}$, comparados a $140 \mathrm{mmHg}$. Entretanto, embora tenha havido diminuição na albuminúria (6,6\% vs. $8,7 \%)$, não se verificou diferença na taxa de DRC terminal. ${ }^{16}$ Em média um paciente hipertenso com nefropatia diabética necessita de 3 a 4 classes de drogas para alcançar o objetivo de controle pressórico. ${ }^{17}$ Existe, porém, a preocupação de não se perseguir alvos de $\mathrm{PA}$ sistólica inferiores a $120 \mathrm{mmHg}$, o que parece se correlacionar com o maior risco de eventos cardiovasculares e óbito.

\section{Prevenção de microalbuminúria com bloqueio do SRAA}

Considerando a elevada incidência de acometimento renal em DM (30-40\%), seria altamente desejável se os efeitos observados do bloqueio do SRAA na nefropatia diabética pudessem ser extrapolados para a prevenção dessa patologia antes de claramente instalada (pacientes normoalbuminúricos). Infelizmente, tanto no DM1 (estudo RASS com enalapril ou $\operatorname{losartan}^{18}$ e estudo DIRECT com candesartan ${ }^{19}$ ), quanto no DM2 (estudo ROADMAP com Olmesartan $^{20}$ e DIRECT ${ }^{19}$ ), o tratamento com drogas bloqueadoras do SRAA não preveniu o aparecimento de microalbuminúria. Aparentemente os efeitos positivos do bloqueio do SRAA ocorrem principalmente em pacientes hipertensos, sendo menos evidentes nos normotensos normoalbuminúricos, que estão na fase inicial da doença.

\section{Há espaço para o duplo bloqueio do SRAA?}

A preocupação de que o resultado do tratamento monoterápico com drogas com ação sobre o SRAA seria subótimo, motivou estratégias de combinação de drogas, visando um bloqueio mais efetivo da angiotensina tecidual. No estudo ONTARGET, ${ }^{21}$ que incluiu milhares de pacientes diabéticos, o tratamento combinado não demonstrou efeito benéfico em eventos cardiovasculares e tampouco efeitos renais superiores aos obtidos com monoterapia. $\mathrm{Na}$ verdade, o tratamento combinado determinou pior desfecho renal, expresso por maior necessidade de diálise e duplicação da creatinina. Esse estudo não incluiu prioritariamente pacientes com nefropatia diabética grave (apenas cerca de $20 \%$ tinham TFG inferior a 60 $\mathrm{ml} / \mathrm{min}$ ou albuminúria). Um estudo menor, realizado na Universidade de São Paulo (USP), ${ }^{22}$ incluiu pacientes com DM2 e nefropatia com macroalbuminúria (TFG médio de $50 \mathrm{ml} / \mathrm{min}$ e albuminúria de $2 \mathrm{~g} / \mathrm{d}$ ). Mesmo nesta população selecionada, o bloqueio duplo (losartan e enalapril) não mostrou superioridade na redução da proteinúria e nem em biomarcadores como VEGF, MCP-1 e RBP.

O bloqueio combinado com um inibidor direto da renina, associado ao losartan (estudo AVOID) trouxe benefício apenas na redução da albuminúria, sem interferir na taxa de perda da função renal. ${ }^{23} \mathrm{O}$ antagonismo da aldosterona, combinado com o bloqueio com IECA ou BRA, também foi testado em estudos menores, com resultados variáveis. Uma metanálise de 11 estudos demonstrou o impacto positivo na redução da proteinúria, embora sem efeito na TFG. ${ }^{24}$

Após estes resultados negativos, principalmente o do estudo ONTARGET, e, além disso, ao elevado risco de hipercalemia, a estratégia do bloqueio duplo foi considerada arriscada e não recomendada. Seu uso eventual deve ser individualizado e reservado para casos excepcionais, sob estrito controle médico.

\section{Albuminúria e progressão da DRC}

Como mencionado, dados recentes têm questionado a validade dos modelos de nefropatia diabética relacionados à albuminúria, prin- 
cipalmente no DM2. ${ }^{25}$ Embora a albuminúria esteja associada classicamente a um prognóstico renal desfavorável em pacientes diabéticos, ela não explica uma parcela de pacientes que desenvolvem perda funcional renal progressiva. Pode-se especular que mecanismos patogênicos distintos podem estar subjacentes a essas diferentes características da doença, determinando inclusive aspectos patológicos diversos e taxas de progressão diferentes. Alguns estudos sugerem que em formas com pouca ou nenhuma albuminúria o comprometimento vascular é predominante. Essas diferenças podem explicar a variabilidade na resposta ao bloqueio do SRAA e indicam que estudos de intervenção na progressão da nefropatia diabética não devem se basear exclusivamente na evolução da albuminúria mas, sim, em desfechos mais "duros", como mortalidade e necessidade de terapia renal substitutiva.

\section{Alvos de controle glicêmico}

A principal evidência sobre os efeitos do controle glicêmico na prevenção de lesões crônicas de órgãos alvos é oriunda do Diabetes Control and Complications Trial (DCCT) e seu seguimento no longo prazo, o Epidemiology of Diabetes Interventions and Complications (EDIC). ${ }^{26}$ Estes estudos demonstraram que o controle da glicemia mais rigoroso (HbA1C $\sim 7 \%)$ resultou em diminuição do risco de nefropatia diabética e que os efeitos persistiram remotamente, mesmo após o controle glicêmico relaxado, determinando queda de $50 \%$ na incidência de necessidade de diálise. Resultados semelhantes, incluindo os efeitos de longo prazo, foram observados no United Kingdom Prospective Diabetes Study (UKPDS). ${ }^{27}$ Essa "memória metabólica" ainda não foi totalmente compreendida, mas reforça a necessidade do controle rigoroso do DM, principalmente em fases iniciais da doença.

Embora o controle glicêmico rigoroso reduza a incidência de microalbuminúria em hipertensos com DM2, nem todos os estudos conseguiram demonstrar benefício do controle metabólico rigoroso $(\mathrm{HbA} 1 \mathrm{C}<7 \%)$ na redução da macroalbuminúria ou na progressão da DRC. Em pacientes com DRC estágio 3 a 5, particularmente naqueles em diálise, existem poucos dados para estabelecer uma recomendação, e embora o controle da $\mathrm{HbA1C}$ entre 7-8\% pareça razoável, deve-se prioritariamente evitar episódios de hipoglicemia.

\section{Novos tratamentos}

$\mathrm{O}$ efeito do stress oxidativo na gênese das complicações do DM, particularmente da nefropatia diabética, abre a perspectiva de intervenções inovadoras. Um antioxidante oral, o bardoxolone, que aumenta a expressão de diversos antioxidantes (catalase, SOD) foi testado em fase II em pacientes com nefropatia diabética em estágio moderadamente avançado. ${ }^{28} \mathrm{Na}$ comparação com placebo, a droga não apenas impediu a queda da TFG como determinou um surpreendente aumento. Esses resultados auspiciosos deram ímpeto ao estudo BEACON, de fase III. Infelizmente, o estudo foi subitamente suspenso em 2012, depois de verificar-se um aumento expressivo de mortalidade no grupo sob tratamento ativo.

A pirfenidona é uma droga anti-inflamatória e antifibrótica licenciada na Europa e Japão para tratamento da fibrose pulmonar. Um pequeno estudo randomizado mostrou proteção da função renal em pacientes diabéticos tratados com pirfenidona, independentemente de alterações nos níveis de albuminúria. ${ }^{29}$ Vitamina $\mathrm{D}$ e análogos desse hormônio têm potencial imunomodulador e podem interferir no SRAA. Um estudo recente, em pacientes sob tratamento com bloqueio do SRAA, utilizou o paricalcitol (análogo da vitamina D) e demonstrou queda de cerca de $20 \%$ na albuminúria basal, quando comparados ao placebo. ${ }^{30}$ Resta saber se esses efeitos serão confirmados e persistentes.

\section{Referências}

1. World Health Organization. World Health Statistics 2012. Geneva; 2012.

2. Remuzzi G, Schieppati A, Ruggenenti P. 
Clinical practice. Nephropathy in patients with type 2 diabetes. N Engl J Med. 2002;346:1145-51.

3. Couser WG, Remuzzi G, Mendis S, Tonelli M. The contribution of chronic kidney disease to the global burden of major noncommunicable diseases. Kidney Int. 2011 Dec;80(12):1258-70. http://www.doi.org/10.1038/ki.2011.368

4. Haffner SM, Lehto S, Ronnemaa T, Pyörälä K, Laakso M. Mortality from coronary heart disease in subjects with type 2 diabetes and in nondiabetic subjects with and without prior myocardial infarction. N Engl J Med. 1998 Jul 23;339(4):229-34.

5. Gerstein HC, Mann JF, Yi Q, Zinman B, Dinneen SF, Hoogwerf B, et al. Albuminuria and risk of cardiovascular events, death, and heart failure in diabetic and nondiabetic individuals. JAMA. 2001 Jul 25;286(4):421-6.

6. Hostetter TH. Chronic kidney disease predicts cardiovascular disease. N Engl J Med. 2004;351(13):1344-6.

7. Eknoyan G, Lameire N, Barsoum R, Eckardt KU, Levin A, Levin N, et al. The burden of kidney disease: improving global outcomes. Kidney Int. 2004 Oct;66(4):1310-4.

8. Bakris GL. Recognition, pathogenesis, and treatment of different stages of nephropathy in patients with type 2 diabetes mellitus. Mayo Clin Proc. 2011 May; 86(5): 444-56. http://www. doi.org/10.4065/mcp.2010.0713

9. Mogensen CE. Diabetes mellitus and the kidney. Kidney Int. 1982 May;21(5):673-5.

10. Gambara V, Mecca G, Remuzzi G, Bertani T. Heterogeneous nature of renal lesions in type II diabetes. J Am Soc Nephrol. 1993 Feb;3(8):145866.

11. Stevens LA, Coresh J, Greene T, Levey AS. Assessing kidney function--measured and estimated glomerular filtration rate. $\mathrm{N}$ Engl J Med 2006 Jun 8;354(23):2473-83.

12. Van Buren PN, Toto R. Hypertension in diabetic nephropathy: epidemiology, mechanisms, and management. Adv Chronic Kidney Dis. 2011 Jan;18(1):28-41. http://www.doi.org/10.1053/j. ackd.2010.10.003

13. Brenner BM, Cooper ME, de Zeeuw D, Keane WF, Mitch WE, Parving $\mathrm{HH}$, et al. Effects of losartan on renal and cardiovascular outcomes in patients with type 2 diabetes and nephropathy. N Engl J Med. 2001 Sep 20;345(12):861-9.

14. Lewis EJ, Hunsicker LG, Bain RP, Rohde RD. The effect of angiotensin-converting-enzyme inhibition on diabetic nephropathy. The Collaborative Study Group. N Engl J Med. 1993 Nov 11;329(20):1456-62.
15. Parving $\mathrm{HH}$, Lehnert $\mathrm{H}$, Brochner-Mortensen J, Gomis R, Andersen S, Arner P, Irbesartan in Patients with Type 2 Diabetes and Microalbuminuria Study Group et al. The effect of irbesartan on the development of diabetic nephropathy in patients with type 2 diabetes. N Engl J Med. 2001 Sep 20;345(12):870-8.

16. ACCORD Study Group, Cushman WC, Evans GW, Byington RP, Goff DC Jr, Grimm RH Jr, Cutler JA, et al. Effects of intensive bloodpressure control in type 2 diabetes mellitus. $\mathrm{N}$ Engl J Med. 2010 Apr 29;362(17):1575-85. http:// www.doi.org/10.1056/NEJMoa1001286

17. Khosla N, Kalaitzidis R, Bakris GL. The kidney, hypertension, and remaining challenges. Med Clin North Am. 2009 May;93(3):697-715, Table of Contents. http://www.doi.org/10.1016/j. mcna.2009.02.001

18. Mauer M, Zinman B, Gardiner R, Suissa S, Sinaiko A, Strand T, et al. Renal and retinal effects of enalapril and losartan in type 1 diabetes. N Engl J Med. 2009 Jul 2;361(1):40-51. http://www.doi.org/10.1056/NEJMoa0808400

19. Bilous R, Chaturvedi N, Sjølie AK, Fuller J, Klein R, Orchard T, et al. Effect of candesartan on microalbuminuria and albumin excretion rate in diabetes: three randomized trials. Ann Intern Med. 2009 Jul 7;151(1):11-20, W3-4.

20. Haller H, Ito S, Izzo JL Jr, Januszewicz A, Katayama S, Menne J, et al. Olmesartan for the delay or prevention of microalbuminuria in type 2 diabetes. N Engl J Med. 2011 Mar 10;364(10):907-17. http://www.doi.org/10.1056/ NEJMoa1007994

21. Ontarget Investigators, Yusuf S, Teo KK, Pogue $\mathrm{J}$, et al. Telmisartan, ramipril, or both in patients at high risk for vascular events. N Engl J Med. 2008;358:1547-59.

22. Titan SM, M Vieira J Jr, Dominguez WV, Barros RT, Zatz R. ACEI and ARB combination therapy in patients with macroalbuminuric diabetic nephropathy and low socioeconomic level: a double-blind randomized clinical trial. Clin Nephrol 2011 Oct;76(4):273-83.

23. Parving $\mathrm{HH}$, Persson F, Lewis JB, Lewis EJ, Hollenberg NK; AVOID Study Investigators. Aliskiren combined with losartan in type 2 diabetes and nephropathy. $\mathrm{N}$ Engl J Med. 2008 Jun 5;358(23):2433-46. http://www.doi. org/10.1056/NEJMoa0708379

24. Navaneethan SD, Nigwekar SU, Sehgal AR, Strippoli GF.Aldosterone antagonists for preventing the progression of chronic kidney disease: a systematic review and meta-analysis. Clin J Am Soc Nephrol 2009 Mar;4(3):542-51. http://www.doi.org/10.2215/CJN.04750908

25. Penno G, Solini A, Bonora E, Fondelli C, Orsi 
E, Renal Insufficiency And Cardiovascular Events (RIACE) Study Group, et al. Clinical significance of nonalbuminuric renal impairment in type 2 diabetes. J Hypertens. 2011 Sep;29(9):802-9. http://www.doi.org/10.1097/ HJH.0b013e3283495cd6

26. DCCT/EDIC Research Group, de Boer IH, Sun W, Cleary PA Lachin JM Molitch ME,et al. Intensive diabetes therapy and glomerular filtration rate in type 1 diabetes. $\mathrm{N}$ Engl J Med. 2011; 365: 2366-76. http://www.doi.org/10.1056/ NEJMoa1111732

27. Gnudi L. Cellular and molecular mechanisms of diabetic glomerulopathy. Nephrology, dialysis, transplantation : official publication of the European Dialysis and Transplant Association - European Renal Association. 2012; 27: 2642-9.

28. Pergola PE, Raskin P, Toto RD, Meyer CJ, Huff JW, Grossman EB, BEAM Study Investigators. et al. Bardoxolone methyl and kidney function in CKD with type 2 diabetes. $N$ Engl J Med. 2011 Jul 28;365(4):327-36. http://www.doi. org/10.1056/NEJMoa1105351

29. Sharma K, Ix JH, Mathew AV, Cho M, Pflueger A, Dunn SR, et al. Pirfenidone for diabetic nephropathy. J Am Soc Nephrol. 2011 Jun;22(6):1144-51. http://www.doi.org/10.1681/ ASN.2010101049

30. de Zeeuw D, Agarwal R, Amdahl M, Audhya P, Coyne D, Garimella T, et al.Selective vitamin $D$ receptor activation with paricalcitol for reduction of albuminuria in patients with type 2 diabetes (VITAL study): a randomised controlled trial. Lancet. 2010 Nov 6;376: 1543-51. http://www.doi.org/10.1016/S01406736(10)61032-X. 